\title{
Oka's inequality for currents and applications
}

\author{
John Erik Fornæss ${ }^{1}$, Nessim Sibony ${ }^{2}$ \\ ${ }^{1}$ Mathematics Department, The University of Michigan, Ann Arbor, MI 48104, USA \\ ${ }^{2}$ Département de Mathématiques, Université Paris Sud, Bâtiment 425 F-91405 Orsay, France
}

Received: 5 June 1993/In revised form: 29 March 1994

\section{Introduction}

In a 1962 paper Oka [Ok] proved that given a family of varieties in an open set $\Omega \subset \mathbb{C}^{2}$ the set $G \subset \Omega$ where the family is normal is pseudoconvex in $\Omega$. The proof is based on the following remarkable inequality.

Let

$$
H=\left\{(|z|<1,|w|<r) \cup\left(r_{1}<|z|<1,|w|<1\right) r_{1}, r<1\right\}
$$

and

$$
\Delta_{\rho}=\{|z|<\rho,|w|<\rho\} .
$$

If $V$ is a closed complex curve in $A_{1}$, then for any $\rho<1$ there exists $C_{p}$ such that

$$
\operatorname{vol}\left(V \cap \Delta_{\rho}\right) \leqq C_{\rho} \operatorname{vol}(V \cap H) .
$$

Here $C_{\rho}$ is independent of $V$.

The result was generalized to varieties of codimension 1 in $\Omega \subset \mathbb{C}^{n}$ by Fujita $[\mathrm{Fu}]$. Riemenschneider [Ri] proved the analogue of inequality $\left({ }^{*}\right)$ for varieties of dimension $p$ in $\Omega \subset \mathbb{C}^{n}$, in which case the standard Hartogs figure has to be replaced by the right analogue. It turns out that the domain $G$ where a family of analytic varieties of dimension $p$, has locally bounded mass is $p$-pseudoconvex (see below for a precise definition) in $\Omega$. This notion was introduced by Rothstein [Ro], see also [Siu2], and developed by Andreotti and Grauert [A-G]. Observe that when a sequence of analytic varieties has bounded volume, then any limit in the Hausdorff metric is an analytic variety, as follows from Bishop's theorem [Bi].

The second author has given in [Si] some estimates on currents from which it is easy to deduce an inequality generalizing $\left({ }^{*}\right)$ to closed positive currents of bidimension $(p, p)$. The purpose in [Si] was to extend the domain of definition of the Monge Ampere operator $\left(d d^{c}\right)^{k}$ to some unbounded plurisubharmonic functions. It was not realized there that the given condition for defining $\left(d d^{c} u\right)^{k}$ 
was that the set where $u$ is locally unbounded is in the $n-k$ pseudoconvex envelope of its complement.

We start here by proving a version of Oka's inequality for currents of the form $u T$, where $T$ is a positive closed current of bidimension $(p, p)$ in $\Omega$ and $u$ is a negative plurisubharmonic function in $\Omega$. It follows from this inequality that $G:=\{z \in \Omega ; u T$ has bounded mass in a neighborhood of $z\}$ is $p$-pseudoconvex in $\Omega$ (usual pseudoconvexity coincides with $n-1$ pseudoconvexity). If the current $(u T)$ has locally bounded mass in $\Omega$ one can define $d d^{c} u \wedge T:=d d^{c}(u T)$.

We then prove a convergence result for the operator $\left(u_{1}, \ldots, u_{q}\right) \mapsto d d^{c} u_{1} \wedge$ $\ldots \wedge d d^{c} u_{q} \wedge T$. The main idea is that if we control the mass or convergence on an open set, then we have the same type of control in the envelope of $l$-pseudoconvexity for the right $l$. When the $\left(u_{j}\right)$ are bounded, this operator was studied by Chern, Levine and Nirenberg [CLN] and Bedford and Taylor [BT]. The case where the $\left(u_{j}\right)$ are unbounded has been considered in Griffiths [Gr], Siu [Siul], Sibony [Si] and more recently by Demailly [De].

Under pseudoconvexity assumptions we can define $T \wedge R_{1} \wedge \ldots \wedge R_{q}$ where $T$ is a current of bidegree $(n-p, n-p)$ and $R_{j}$ are currents of bidegree $(1,1)$. When the currents are in $\mathbb{P}^{k}$ we prove a Bezout type theorem i.e. express the mass of $T \wedge \ldots \wedge R_{q}$ in terms of the mass of the factors.

Let $T$ be a positive closed current in $\mathbb{P}^{k}$ of bidegree $(1,1)$. So locally $T$ can be written as $d d^{c} u$. Assuming that $u$ is continuous, we show that support $T^{l}$ is connected, provided $2 l \leqq k$.

In the last paragraph we apply the results on currents to holomorphic dynamics in $\mathbb{P}^{k}$. This was the main motivation to try to develop some tools in order to understand closed currents which are not analytic varieties. To a holomorphic surjective map $f: \mathbb{P}^{k} \mapsto \mathbb{P}^{k}$ of degree $d>1$, one associates a positive closed current $T$ of bidegree $(1,1)$. The support of $T^{l}:=T \wedge \ldots \wedge T$ ( $l$ factors) are of dynamical interest. In particular the support of $T$ coincides with the Julia set $J_{0}$ of $f$ : the sequence $\left(f^{n}\right)$ is equicontinuous precisely on $\mathbb{P}^{k} \backslash J_{0}$. In this context we show that if $2 l \leqq k$, then support $T^{l}$ is connected.

\section{Oka's inequality}

We first define the notion of $k$-pseudoconvexity, see [Ri]. Let $0<r_{1}^{\prime}<r^{\prime}$ and $0<r_{1}<r$.

Definition 2.1 An $(\boldsymbol{n}-\boldsymbol{k}, \boldsymbol{k})$ Hartogs figure $H$ is defined as

$$
\begin{aligned}
H=\{ & \left\{(z, w) ; z \in \mathbb{C}^{n-k}, w \in \mathbb{C}^{k},\|z\|<1,\|w\|<r\right\} \\
& \cup\left\{(z, w) ; z \in \mathbb{C}^{n-k}, w \in \mathbb{C}^{k}, r_{1}<\|z\|<1,\|w\|<1\right\}
\end{aligned}
$$

where $0<r_{1}, r<1$. We set

$$
\hat{H}=\left\{(z, w) ; z \in \mathbb{C}^{n-k}, w \in \mathbb{C}^{k},\|z\|<1,\|w\|<1\right\}=\Delta^{n} .
$$

Here $\|z\|=\max \left|z_{j}\right|,\|w\|=\max \left|w_{j}\right|$. 
Definition 2.2 ( $k$-pseudoconvexity) Let $\Omega_{0} \subset \Omega$ be open subsets of $\mathbb{C}^{n}, 0<$ $k<n$. Then $\Omega_{0}$ is $\boldsymbol{k}$-pseudoconvex in $\Omega$ if it satisfies the Kontinuitätssatz with respect to $(n-k)$ polydiscs. More precisely, whenever $H$ is an $(n-k, k)$ Hartogs figure, $\Phi: \hat{H} \mapsto \Omega$ is a $1-1$ holomorphic map and $\Phi(H) \subset \Omega_{0}$, then $\Phi(\hat{H}) \subset \Omega_{0}$.

Usual pseudoconvexity is the same as $(n-1)$ pseudoconvexity.

For $\rho>0, \Delta_{\rho}$ will denote the polydisc of radius $\rho$.

Theorem 2.3 [Ri] Let $X$ be a pure $k$-dimensional closed complex analytic subvariety of the unit polydisc $\Delta \subset \mathbb{C}^{n}, 0<k<n$. Then if $0<\rho<1$, and $H$ is an $(n-k, k)$ Hartogs figure, $\operatorname{vol}\left(X \cap \Delta_{\rho}\right) \leqq C_{\rho} \operatorname{vol}(X \cap H)$ for $C_{\rho}$ independent of $X$.

It follows that if $\left(X_{i}\right)$ is a family of closed analytic varieties of pure dimension $k$ in $\Omega \subset \mathbb{C}^{n}$, then if $\Omega^{\prime}:=\left\{z ; \exists \delta>0 ; \sup _{t} \operatorname{vol}\left(X_{t} \cap B(z ; \delta)\right)<\infty\right\}$ then $\Omega^{\prime}$ is $k$-pseudoconvex in $\Omega$.

We want to prove a version of the previous theorem for positive closed currents or even for currents of the form $u T$ where $T$ is a positive closed current and $u$ is a plurisubharmonic function.

For the fundamental results on currents we refer to [de Rh], [Lel] or [LG]. We recall a few facts.

Let $\Omega$ be an open set in $\mathbb{C}^{n}$. Denote by $D^{p, q}(\Omega)$ the space of smooth differential forms of bidegree $(p, q)$ with compact support in $\Omega$. The space of currents of bidimension $(p, q)$, hence of bidegree $(n-p, n-q)$ is the dual space of $D^{p, q}(\Omega)$. A current $T$ of bidimension $(p, p)$ and bidegree $(n-p, n-$ $p)$ is positive if for all $\alpha_{1}, \ldots, \alpha_{p} \in D^{1,0}(\Omega)$ the current $T \wedge i \alpha_{1} \wedge \overline{\alpha_{1}} \wedge \ldots \wedge$ $i \alpha_{p} \wedge \overline{\alpha_{p}}$ is a positive distribution. A current $U$ is negative if $-U$ is positive.

If $\alpha$ is a $k$-covector in $\mathbb{C}^{n} \simeq \mathbb{R}^{2 n}$, let $|\alpha|$ denote the usual Hilbertian norm of $\alpha$. If $T$ is a current of bidimension $(p, p)$ of order zero, i.e. with measure coefficients, we define the measure $M_{V}[T]$ on $\Omega$ as follows. If $V$ is open in $\Omega$, let $M_{V}[T]=\sup \left\{|T(\Phi)| ; \Phi \in D^{p, p}(V),|\Phi(x)| \leqq 1, x \in V\right\}$. This is called the mass norm of $T$.

Let $d^{c}:=(i / 2 \pi)(\bar{\partial}-\hat{\partial})$, then $d d^{c}=(i / \pi) \hat{\partial} \bar{\partial}$. Let $\beta:=d d^{c}|z|^{2}$. If $T$ is a positive current of bidimension $(p, p)$, then the trace measure $\sigma_{T}$ is defined as $\sigma_{T}:=T \wedge \beta^{p} / p$ !. It is easy to show that there exists a constant $C$, depending only on $n$ and $p$ such that for every open set $V \subset \Omega$,

$$
(1 / C) \sigma_{r}(V) \leqq M_{V}[T] \leqq \sigma_{T}(V)
$$

An upper semicontinuous function $u: \Omega \mapsto[-\infty, \infty)$ is plurisubharmonic if and only if $u \in L_{\mathrm{loc}}^{1}(\Omega)$ and $d d^{c} u \geqq 0$, for short we will say that $u$ is p.s.h.

Theorem 2.4 Let $H$ be an $(n-l, l)$ Hartogs figure. Assume $\hat{H}=\Delta$. For every $\rho<1$, there exists a constant $C_{p}$ such that for every negative current $U$ in a neighborhood of $\overrightarrow{\bar{A}}$, of bidimension $(l, l)$, such that $d d^{c} U \geqq 0$ we have

$$
M_{\Delta_{p}}[U]+M_{\Delta_{p}}\left[d d^{c} U\right] \leqq C_{\rho} M_{H}[U] .
$$


Proof. Let $\Omega$ be an open set in $\mathbb{C}^{n}$. Assume $\omega \Subset \tilde{\omega} \Subset \Omega$, and that $\omega=\{z ; z \in$ $\tilde{\omega}, \psi(z)<0, \nabla \psi \mid \partial \omega \neq 0\}$ for some smooth function $\psi$ defined on $\tilde{\omega}$. Let $M$ be a closed subset in $\Omega$. Let $x \in M \cap \omega$ and let $\phi \in C^{\infty}(\Omega)$ such that

(i) $\phi(x)>0$

(ii) $\phi \equiv 0$ on a neighborhood $V$ of $\partial \omega \cap M$.

Let $h$ be a smooth function in $\Omega, 0 \leqq h \leqq 1, h=1$ in a neighborhood of $\omega$.

We will need the following lemma. See also Lemma 4.1 in [Si].

Lemma 2.5 Let $\omega, \Omega, \phi, h$ be as defined above. Let $S$ and $U$ be currents with smooth coefficients in $\Omega$. Assume $S$ is closed of bidegree $(p, p)$ and $U$ is of bidegree $(q, q)$ with $p+q=n-1$. Then we have

(1) $\int_{(1)} \phi(1-h) d d^{c} U \wedge S+\int_{\omega}(-U) \wedge d d^{c} \phi \wedge S=\int_{()}(-U) \wedge d d^{c}(\phi h) \wedge S$.

If $U \leqq 0, d d^{c} U \geqq 0$ then relation (1) holds without smoothness assumption on $U$.

Proof. When $\chi$ is a smooth function with compact support then since $S$ is closed

$$
\int \chi d d^{c} U \wedge S=\int d d^{c} \chi \wedge U \wedge S
$$

So we just apply the above formula with $\chi=\phi(1-h)$ which is of compact support since $h=1$ near $\partial \omega$.

If $U$ is not smooth, let $U_{\varepsilon}$ be regularized currents, $U_{\varepsilon}$ is just convolution of $U$ with an approximation of identity. Relation (1) holds for $U_{\varepsilon}$. We can just let $\varepsilon \mapsto 0$.

The formula of integration by part is written in the form (1) to emphasize that if $h=0$ near $x$ and if $d d^{c} \phi \wedge S$ is positive then it is posible to control the mass of $d d^{c} U$ near $x$ and of $(-U) \wedge d d^{c} \phi \wedge S$ by what happens near the support of $d d^{c}(\phi h)$.

We continue the proof of the Theorem. Let $\Omega$ be a neighborhood of $\vec{\Delta}$ where $U$ is defined. Recall that $H=\left\{(z, w), z \in \mathbb{C}^{n-l}, w \in \mathbb{C}^{l},\|z\|<1,\|w\|<\right.$ $\left.r_{1}\right\} \cup\left\{r_{1}^{\prime}<\|z\|<1,\|w\|<1\right\}$. Let $M=\Delta \backslash H$. Let $M_{1}$ be the set of points in $\Delta_{p_{1}} \backslash H$ for some $\rho<\rho_{1}<1$, such that exactly one of the $w$-coordinates is $\geqq r_{1}$. Let $x \in M_{1}$. For simplicity assume that $\left|x_{n-l+1}\right|=\left|w_{1}\right| \geqq r_{1}$ and $\left|x_{n-1+2}\right|$ $=\left|w_{2}\right|<r_{1}, \ldots,\left|x_{n}\right|=\left|w_{l}\right|<r_{1}$. Let $\phi=1 /\left|w_{1}\right|^{2}$. Let $H_{1}$ be the $(n-l, 1)$ Hartogs figure obtained by fixing the coordinates $w_{2}=x_{n-l+2}, \ldots, w_{l}=x_{n}$, with the same numbers $r_{1}, r_{1}^{\prime}$. We can construct an open set $\omega_{1}$ in $\mathbb{C}^{n-l+1}$ such that $\phi(x)>\max _{\hat{c}()_{1} \cap\left[\hat{H}_{1} \backslash H_{1}\right]} \phi$. Fatten $\omega_{1}$ to obtain an open set $\omega$ in $\mathbb{C}^{n}$ and extend $\phi$ to $\Phi_{1}$ such that $\Phi_{1}(x)>\max _{\hat{c} \omega \cap[\hat{H} \backslash H]} \Phi_{1}$, by adding to the trivial extension a function $\theta$ of $w_{2}, \ldots, w_{n}$ only.

Let $\Phi_{2}:=\Phi_{1}+\delta|(z, w)|^{2}$. For $\delta>0$ small enough, $\Phi_{2}(x)>\sup _{\hat{c} \omega \cap} \Phi_{2}$ $:=c$. But also $d d^{c} \Phi_{2} \wedge\left(d d^{c}\left|w^{\prime}\right|^{2}\right)^{l-1}>0$ where $w^{\prime}=\left(w_{2}, \ldots, w_{l}\right)$. This is 
because the negativity in $d d^{c} \Phi_{2}$ introduced by the function $\theta\left(w^{\prime}\right)$ is cancelled by $\left(d d^{c}\left|w^{\prime}\right|^{2}\right)^{1-1}$.

Let $\lambda$ be a smooth increasing convex function on $[0, \infty)$, vanishing on a neighborhood of $[0, c]$ and then strictly increasing, we can assume that $\lambda\left(\Phi_{2}(x)\right)>0$. Let $\Phi=\lambda \circ \Phi_{2}$. We apply Lemma 2.5 with $S=\left(d d^{c}\left|w^{\prime}\right|^{2}\right)^{l-1}$ and $\phi=\Phi$ and $h$ a function vanishing near $x, h$ has value 1 near $\partial \omega$, but $M \cap$ support $h$ is contained in $(\phi=0)$.

Relation (1) in Lemma 2.5 gives an estimate of $d d^{c} U \wedge\left(d d^{c}\left|w^{\prime}\right|^{2}\right)^{l-1}$ and of $(-U) \wedge d d^{c} \phi \wedge S$ near $x$ in terms of the mass of $U$ on a compact set of $H$. If we apply the same argument to suitable perturbations of the coordinates $w$, we finally get an estimate of the mass of $(-U)$ and $d d^{c} U$ near $x$ in terms of quantities supported in a compact set of $H$. We have proved the theorem for $M_{1}$ instead of $A_{\rho}$. Let $M_{j}$ be the set of points in $\Delta_{\rho j} \backslash H$ such that exactly $j$ of the $w$-coordinates are $\geqq r_{1}$ where we have chosen $1>\rho_{1}>\cdots>\rho_{l}=\rho$. We will prove the theorem by induction in $j$. For simplicity of notation we prove it for $j=2$. Let $x \in M_{2}$. We can assume that $\left|x_{n-l+1}\right|=\left|w_{1}\right| \geqq r_{1}$ and $\left|x_{n-l+2}\right|=\left|w_{2}\right| \geqq r_{1}$ while $\left|w_{3}\right|<r_{1}, \ldots,\left|w_{l}\right|<r_{1}$.

Let $\phi_{2}=1 /\left|w_{2}\right|^{2}$. We can construct an open set $\omega_{2}$ in $\mathbb{C}^{n-l+1}$ such that $\phi_{2}(x)>\max _{\tilde{c} \omega_{2} \cap\left[\hat{H}_{2} \backslash H_{2}\right]} \phi$. We fatten $\omega_{2}$ to obtain an open set $\omega$ in $\mathbb{C}^{n}$ and proceed as above. We will obtain an estimate of the mass of $U$ and $d d^{c} U$ near $x$ in term of the mass in $H \cup M_{1}$ which in turn is controlled by the mass in $H$. So by induction we get the estimate in the theorem.

Corollary 2.6 Let $\left(U_{1}\right)$ be a sequence of negative currents of bidimension $(p, p)$ in a complex manifold $\Omega$. Assume for all $i, d d^{c} U_{1} \geqq 0$. Then the domain $\Omega_{0}$ on which the family has locally bounded mass is p-pseudoconvex. In particular, if $U$ is a negative current of bidimension $(p, p)$ in $\Omega$, such that $d d^{c} U \geqq 0$, then $\Omega \backslash(\operatorname{supp} U)$ is p-pseudoconvex in $\Omega$. If $T$ is a positive closed current of bidimension $(p, p)$ on $\Omega$, then $\Omega \backslash \operatorname{supp} T$ is $p$ pseudoconvex.

Proof. Let $H$ be an $(n-p, p)$ Hartogs figure and $f: \hat{H} \rightarrow \Omega$ be an injective holomorphic map. If $f(H) \subset \Omega_{0}$, Theorem 2.4 shows that $f(\hat{H}) \subset \Omega_{0}$. The estimate in the theorem is just given with $f=\mathrm{Id}$. So $\Omega_{0}$ is $p$-pseudoconvex in $\Omega$. To show that $\Omega \backslash \operatorname{supp} U$ is $p$-pseudoconvex we just apply the first part of the corollary to the sequence of currents $U_{k}=k U, k \in \mathbb{N}$. If $T$ is positive and closed, we apply the previous result to $U=-T$.

Let $T$ be a positive closed current of bidimension $(p, p)$ in $\Omega$. Let $u$ be a plurisubharmonic function on $\Omega$. We consider the closed set $M(T, u)=\{q ; q \in$ $\Omega, u T$ is not of finite mass in any neighborhood of $q\}$. For simplicity, since the results are of semi local nature (in neighborhoods of compacts) we will assume that $u<0$.

Proposition 2.7 Let $T, u, \Omega$ be as above. Then $\Omega \backslash M(T, u)$ is p-pseudoconvex. And on $\Omega \backslash M(T, u)$ the current $u T$ is negative and satisfies $d d^{c}(u T) \geqq 0$. 
Proof. Let $H$ be an $(n-p, p)$ Hartogs figure, assume $\hat{H}=\Delta \Subset \Omega$. Let $u_{j} \searrow u$ be a decreasing sequence of smooth plurisubharmonic functions in a neighborhood of $\bar{\Delta}$. If $U_{j}:=u_{j} T$ we clearly have $U_{j} \leqq 0$ for large $j$ and $d d^{c} U_{j} \geqq 0$. Theorem 2.4 implies that

$$
\int_{A_{\rho}}\left|u_{j}\right| \sigma_{T} \leqq C_{\rho} \int_{H}\left|u_{j}\right| \sigma_{T} \leqq C_{\rho} \int_{H}|u| \sigma_{T} .
$$

Here $\sigma_{T}$ denotes the trace measure for the current $T$. Hence if $\int_{H}|u| \sigma_{T}<\infty$ we get that

$$
\int_{A_{\rho}}|u| \sigma_{T} \leqq C_{\rho} \int_{H}|u| \sigma_{T},
$$

by Lebesgue's dominated convergence theorem. The $p$-pseudoconvexity of $\Omega \backslash M(T, u)$ follows easily from the above estimate. Since locally in $\Omega \backslash M(T, u)$, $u_{j} T \mapsto u T$ in the sense of currents and since $d d^{c}\left(u_{j} T\right)=d d^{c} u_{j} \wedge T \geqq 0$ we get that $d d^{c}(u T) \geqq 0$.

\section{The operator $\left(u_{1}, \ldots, u_{q}\right) \mapsto d d^{c} u_{1} \wedge \ldots \wedge d d^{c} u_{q} \wedge T$}

It is very useful, in many questions in Algebraic Geometry and Complex Analysis, to define an expression such that $d d^{c} u \wedge T$ where $T$ is a closed $(p, p)$ current and $u$ is an unbounded plurisubharmonic function. The case where $u$ is bounded is studied in [BT].

Here we want to extend the approach in [Si] to define

$$
d d^{c} u_{1} \wedge \ldots \wedge d d^{c} u_{k} \wedge T
$$

under quite general assumptions on $u_{1}, \ldots, u_{k}$ and $T$.

Let $\Omega$ be an open subset in $\mathbb{C}^{n}$. If $u$ is a plurisubharmonic function in $\Omega$, define $M(u):=\{q ; q \in \Omega, u$ is unbounded in any neighborhood of $q\}$. Recall that if $T$ is a positive closed $(p, p)$ current we define $M(T, u):=\{z ; z \in \Omega, u$ is not $\sigma_{T}$ integrable in a neighborhood of $\left.q\right\}$. In $\Omega \backslash M(T, u)$ we will define $d d^{c} u \wedge T:=d d^{c}(u T)$.

If $X$ is a closed set in $\Omega$, we say that $X$ is in the envelope of $p$ pseudoconvexity of $\Omega \backslash X$ with respect to $\Omega$ if all points in $X$ can be reached by pushing polydiscs of dimension $(n-p)$ using biholomorphic images of $(n-p, p)$ Hartogs figures with hulls in $\Omega$. So we use the same procedure as for obtaining the envelope of holomorphy by pushing one dimensional discs.

Proposition 3.1 Let $u$ be a plurisubharmonic function in $\Omega$. And let $T$ be a positive closed bidimension $(p, p)$ current on $\Omega$. If $M(u) \cap \operatorname{supp} T:=X$ is in the envelope of p-pseudoconvexity of $\Omega \backslash X$ with respect to $\Omega$, then $u$ is locally $\sigma_{T}$ integrable.

Proof. Without loss of generality we can assume $u<0$ on $\Omega$. It is clear that $M(T, u) \subset M(u) \cap \operatorname{supp} T$. Since as proved in Proposition $2.7 \Omega \backslash M(T, u)$ is $p$-pseudoconvex it follows that $M(T, u)$ is empty. 
Proposition 3.2 Let $u_{j}, u$ be nonpositive plurisubharmonic functions in $\Omega$. Let $T_{j}, T$ be positive closed currents of bidimension $(p, p)$ in $\Omega$. Assume

(1) $T_{j} \mapsto T$,

(2) $\left(u_{j} T_{j}\right)$ has uniformly bounded mass on every compact,

(3) $u_{j} \mapsto u$ in $L_{\mathrm{loc}}^{1}$.

If $L$ is any weak limit of $u_{j} T_{j}$, then $L \leqq u T$. If $u_{j} \geqq u$ and $T_{j} \leqq T$, then $u_{j} T_{j} \mapsto u T$.

Proof. Let $\gamma \geqq 0$ be a smooth form with compact support $X \subset \Omega$ of bidegree $(p, p)$. Let $f$ be a continuous function on $X$ such that $f \geqq u$ on $X$. By Hartogs lemma, see $[\mathrm{H}]$,

$$
\varlimsup_{j} \sup _{X}\left(u_{j}-f\right) \leqq \sup _{X}(u-f) \leqq 0 .
$$

Hence given $\varepsilon>0$, there is a $j_{0}$ so that for $j \geqq j_{0}, u_{j} \leqq f+\varepsilon$. Since $\gamma \geqq 0$ and $T_{j} \geqq 0$ we have

$$
u_{j} T_{j} \wedge \gamma \leqq(f+\varepsilon) T_{j} \wedge \gamma
$$

Hence

$$
L \wedge \gamma \leqq(f+\varepsilon) T \wedge \gamma .
$$

Since $\varepsilon$ and $f$ are arbitrary we get that $L \leqq u T$. If $u_{\jmath} \geqq u$ and $T_{j} \leqq T$. we can assume using again Hartogs lemma that $u$, $\leqq 0$ on support $\gamma$, we have

$$
\int u T \wedge \gamma \leqq \int u_{j} T \wedge \gamma \leqq \int u_{j} T_{1} \wedge \gamma \mapsto L \wedge \gamma .
$$

So $L=u T$.

Corollary 3.3 Let $u_{n} \leqq 0$ be plurisubharmonic in $\Omega$ and assume that $u_{n} \mapsto u$ in $L_{\text {loc }}^{1}$ and that $u_{n} \geqq u$. Then for any positive closed current of bidimension $(p, p)$, we have in $\Omega \backslash M(T, u)$ that $u_{n} T \mapsto u T$ and that $d d^{c} u_{n} \wedge T \mapsto d d^{c} u \wedge T$.

Proof. Since $u_{j} \leqq 0,\left|u_{j}\right| \leqq|u|$ so given a compact $K$ in $\Omega \backslash M(T, u)$,

$$
\int_{K}\left|u_{j}\right| d \sigma_{T} \leqq \int_{K}|u| d \sigma_{T}
$$

Hence we can apply Proposition 3.2.

Let $\Lambda^{x}$ denote the Hausdorff measure of dimension $\alpha$. We have the following convergence result.

Corollary 3.4 Let $\left(u_{1}\right)$ be a sequence of plurisubharmonic functions in $\Omega$. Assume $u_{j} \mapsto u$ in $L_{\mathrm{loc}}^{1}$ and $u_{j} \geqq u$. Let $T$ be a positive, closed current of bidimension $(p, p)$ in $\Omega$. If $\Lambda^{2 p}(M(u) \cap \operatorname{supp} T)=0$, then $u_{j} T \mapsto u T$ in $\Omega$.

Proof. We just have to check that $M(T, u)$ is empty. It is clear that $M(T, u) \subset$ $M(u) \cap \operatorname{Supp} T:=E$. The idea is to show that $E$ is in the $p$ envelope of the complement. By the results of Federer [Fe], for almost all $n-p$ planes say parallel to a given one the intersection with $E$ is empty, hence we can construct Hartogs figures of the right type. More precisely, fix $x \in E$. Since 
$A^{2 p}(E \backslash\{x\})=0$, almost every $n-p$ complex plane through $x$ does not intersect $E \backslash\{x\}$. Assume that $x=0$ and let $L=\left\{w_{1}=\ldots w_{p}=0\right\}$ be such a plane. Let $\left(z_{1}, \ldots, z_{n-p}\right)$ be the coordinates in $L$. Fix a polydisc $\Delta^{n-p} \Subset \Omega$ centered at the origin. Since $\partial \Delta^{n-p} \cap E=\varnothing$, we can find a polydisc $\Delta^{p}$ in the orthogonal complement $L^{\perp}$ centered at the origin such that $\left(\partial \Delta^{n-p} \times \Delta^{p}\right) \cap E=\varnothing$. We can then complete the Hartogs figure $H \subset \Omega \backslash E$ such that $0 \in \hat{H}$.

Theorem 3.5 Let $T$ be a closed positive current of bidimension $(p, p)$ in $\Omega, 0<p<n$. Let $u_{1}, \ldots, u_{q}$ be plurisubharmonic $\leqq 0$ functions in $\Omega$. If for all $j_{1}, \ldots, j_{m}, M\left(u_{j_{1}}\right) \cap \ldots \cap M\left(u_{j_{m}}\right) \cap \operatorname{supp} T$ is in the $p-m+1$ envelope of pseudoconvexity of the complement, then $u_{1} d d^{c} u_{2} \wedge \ldots \wedge d d^{c} u_{q} \wedge T$ has locally bounded mass in $\Omega$ and similarly for $d d^{c} u_{1} \wedge \ldots \wedge d d^{c} u_{q} \wedge T$. Moreover the mass on a compact set of $\Omega$ is majorized by the mass on a compact where all the $u_{j}$ are bounded. We also have $u_{1}^{j} d d^{c} u_{2}^{j} \wedge \ldots \wedge d d^{c} u_{q}^{j} \wedge T \mapsto$ $u_{1} d d^{c} u_{2} \wedge \ldots \wedge d d^{c} u_{q} \wedge T$ provided $u_{k}^{j} \mapsto u_{k}$ in $L_{\text {loc }}^{1}$ and $u_{k}^{j} \geqq u_{k}$, moreover $d d^{c} u_{1}^{j} \wedge \ldots \wedge d d^{c} u_{q}^{j} \wedge T$ converge weakly to $d d^{c} u_{1} \wedge \ldots \wedge d d^{c} u_{q} \wedge T$.

Proof. Case $q=1$. We assume $M(u) \cap \operatorname{supp} T$ is in the $p$-convex envelope of the complement relative to $\Omega$. Then $u T$ is well defined in $\Omega$ and if $L$ is a compact in $\Omega$, Theorem 2.4 shows that

$$
\int_{L}|u| d \sigma_{T} \leqq c \int_{K}|u| d \sigma_{T}
$$

where $K$ is a compact of $\Omega \backslash M(u)$. Let $\chi$ be a nonnegative test function with value 1 in a neighborhood of $L$. If $\beta=d d^{c}|z|^{2}$, then

$$
M_{L}\left[d d^{c} u \wedge T\right] \leqq \int \chi d d^{c} u \wedge T \wedge \beta^{s}=\int u T \wedge \beta^{s} \wedge d d^{c} \chi .
$$

So we also get that $M_{L}\left[d d^{c} u \wedge T\right] \leqq c^{\prime} M_{K^{\prime}}[u T]$. For $q=1$ the convergence result is just Corollary 3.3.

So assume the theorem has been proved for $(q-1)$ functions $\left(v_{j}\right)$. Let $S=d d^{c} u_{2} \wedge \ldots \wedge d d^{c} u_{q} \wedge T$ which is, by induction, a well defined current on $\Omega$ of bidimension $(p-q+1, p-q+1)$. We want to show that $\Omega \backslash M\left(u_{1}, S\right)$ contains the complement of $M\left(u_{1}\right) \cap \ldots \cap M\left(u_{q}\right) \cap \operatorname{supp} T$. Since $\Omega \backslash M\left(u_{1}, S\right)$ is $p-q+1$ pseudoconvex, the hypotheses of the theorem imply that $M\left(u_{1}, S\right)$ is empty.

Fix $z_{0} \notin M\left(u_{1}\right) \cap \ldots \cap M\left(u_{q}\right) \cap \operatorname{supp} T$. If $u_{1}$ is bounded near $z_{0}$ we are done. Assume $z_{0}=0$ and that $u_{2}$ is bounded on a neighborhood of $\bar{B}:=\bar{B}(0, r)$. Replacing $u_{2}$ by $\max \left(u(z), A\left(|z|^{2}-r^{2}\right)\right)$ we can assume that $u_{2}$ is unchanged on $B_{1}=B(0, r / 4)$, and is equal to $A\left(|z|^{2}-r^{2}\right)$ in a neighborhood of $\partial B$. Let $T^{\prime}=d d^{c} u_{3} \wedge \ldots \wedge d d^{c} u_{q} \wedge T$. We will show that

$$
\int_{B}-u_{1} d d^{c} u_{2} \wedge T^{\prime} \wedge \beta^{s}
$$

is bounded by the mass of $u_{1} T^{\prime}$ on $B$. 
Let $h$ be a smooth function supported near $\partial B$ with value 1 near $\partial B$. Let $v_{1}^{j} \searrow u_{1}, v_{1}^{j}$ smooth plurisubharmonic functions. We can apply Lemma 2.5 with $U=v_{1}^{j} d d^{c} u_{2} \wedge T^{\prime}$ and $S=\beta^{s-1}$ (in this case $\omega=B, \phi=|z|^{2}$ and $M$ is empty). We have

$$
\begin{gathered}
\int_{B} \phi(1-h) d d^{c} v_{1}^{J} \wedge d d^{c} u_{2} \wedge T^{\prime} \wedge \beta^{s-1} \\
-\int_{B} v_{1}^{j} d d^{c} \phi \wedge d d^{c} u_{2} \wedge T^{\prime} \wedge \beta^{s-1} \\
=-\int_{B} v_{1}^{j} d d^{c}(\phi h) \wedge d d^{c} u_{2} \wedge T^{\prime} \wedge \beta^{s-1}
\end{gathered}
$$

Observe that $-v_{1}^{J} \geqq 0$ and $-A^{\prime} \beta \leqq d d^{c}(\phi h) \leqq A^{\prime} \beta$ and that on the support of $h, d d^{c} u_{2}=A \beta$. So the last integral is smaller in absolute value than

$$
-A A^{\prime} \int_{B} v_{1}^{j} T^{\prime} \wedge \beta^{s+1}
$$

Using Fatou's lemma and the induction hypothesis we get

$$
\int_{B}\left|u_{1}\right| d d^{c} \phi \wedge d d^{c} u_{2} \wedge T^{\prime} \wedge \beta^{s-1} \leqq A A^{\prime} \int_{B}\left|u_{1}\right| T^{\prime} \wedge \beta^{s+1}
$$

Hence we have shown $\left(^{*}\right)$. As a consequence, also the mass of $d d^{c} u_{1} \wedge$ $d d^{c} u_{2} \wedge T^{\prime}$ on $B_{1}$ is bounded by the mass of $u_{1} T^{\prime}$ on $B$.

We now turn to the convergence question. Let $u_{1}^{J} \geqq u_{1}, \ldots, u_{q}^{\prime} \geqq u_{q}$. We know that if $R$ is any limit point of $u_{1}^{\prime} d d^{c} u_{2}^{\prime} \wedge \ldots \wedge T$ then $R \leqq u_{1} d d^{c} u_{2} \wedge$ $\ldots \wedge T$ (by Proposition 3.2). In order to prove equality, it is enough to prove that for any $x_{0}$ there exists an open set $\omega \Subset \Omega, x_{0} \in \omega$, a smooth function $\phi$ in a neighborhood of $\bar{\omega}$, a positive closed form $\gamma$ such that $d d^{c} \phi \wedge \gamma$ is strictly positive in a neighborhood of $x_{0}$ and $\int_{(1)} u_{1} d d^{c} u_{2} \ldots \wedge T \wedge \gamma \wedge d d^{c} \phi \leqq$

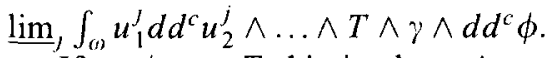

If $x_{0} \notin \operatorname{supp} T$ this is clear. Assume $x_{0} \notin M\left(u_{1}\right) \cap \ldots \cap M\left(u_{q}\right)$. Then there exists a ball $B$ such that $\left(u_{l}^{j}\right)$ is a bounded sequence in a neighborhood of $\bar{B}$ for some $1 \leqq l \leqq q$. Let $B=\{\psi<0\}, \psi$ plurisubharmonic. Since the result we want is local we can assume that on a neighborhood $\omega_{1}$ of $\partial B, u_{l}^{i}=A \psi$ where $A$ is a large constant. Let $h$ be a function in $C_{0}^{\infty}\left(\omega_{1}\right)$ with value 1 in a neighborhood of $\partial B$. We are going to use repeatedly the identity of Lemma 2.5 with $\phi=|z|^{2}$ and $M$ empty.

Assume that all the $u_{k}^{\prime} \mathrm{s}$ are smooth.

Let $T^{\prime}$ denote $T \wedge \beta^{p-q}$, we have

$$
\begin{aligned}
& \int_{B} u_{1} d d^{c} u_{2} \wedge \ldots \wedge T^{\prime} \wedge d d^{c} \phi \\
& \leqq \\
& \int_{B} u_{1}^{\prime} d d^{c} u_{2} \wedge \ldots \wedge T^{\prime} \wedge d d^{c} \phi \\
& =\int_{B} \phi(1-h) d d^{c} u_{1}^{\prime} \wedge d d^{c} u_{2} \wedge \ldots \wedge T^{\prime} \\
& \quad+\int_{B} u_{1}^{j} d d^{c}(\phi h) \wedge d d^{c} u_{2} \wedge \ldots \wedge T^{\prime}
\end{aligned}
$$




$$
\begin{aligned}
= & \int_{B} u_{2} d d^{c} \phi \wedge d d^{c} u_{1}^{j} \wedge \ldots \wedge T^{\prime} \\
& -\int_{B} u_{2} d d^{c}(\phi h) \wedge d d^{c} u_{1}^{j} \wedge \ldots \wedge T^{\prime} \\
& +\int_{B} u_{1}^{j} d d^{c}(\phi h) \wedge d d^{c} u_{2} \wedge \ldots \wedge T^{\prime} \\
\leqq & \int_{B} u_{2}^{j} d d^{c} \phi \wedge d d^{c} u_{1}^{j} \wedge T^{\prime} \ldots \\
& -\int_{B} u_{2} d d^{c}(\phi h) \wedge d d^{c} u_{1}^{j} \wedge \ldots \wedge T^{\prime} \\
& +\int_{B} u_{1}^{j} d d^{c}(\phi h) \wedge d d^{c} u_{2} \wedge \ldots \wedge T^{\prime} \ldots=
\end{aligned}
$$

(where we do not write terms containing $d d^{c}(\phi h)$ )

$$
\begin{aligned}
= & \int_{B} \phi(1-h) d d^{c} u_{1}^{j} \wedge d d^{c} u_{2}^{j} \wedge d d^{c} u_{3} \wedge \ldots \\
& +\int_{B} u_{2}^{j} d d^{c}(\phi h) \wedge d d^{c} u_{1}^{j} \wedge \ldots T^{\prime}+\ldots \\
= & \int_{B} u_{1}^{j} d d^{c} u_{2}^{j} \wedge d d^{c} u_{3} \wedge \ldots \wedge T^{\prime} \wedge d d^{c} \phi \\
& -\int_{B} u_{1}^{j} d d^{c}(\phi h) \wedge d d^{c} u_{2}^{j} \wedge \ldots \wedge T^{\prime} \\
& +\int_{B} u_{2}^{j} d d^{c}(\phi h) \wedge d d^{c} u_{1}^{j} \wedge \ldots \wedge T^{\prime} \\
& -\int_{B} u_{2} d d^{c}(\phi h) \wedge d d^{c} u_{1}^{j} \wedge \ldots \wedge T^{\prime} \\
& +\int_{B} u_{1}^{j} d d^{c}(\phi h) \wedge d d^{c} u_{2} \wedge \ldots \wedge T^{\prime} \\
\leqq & \int_{B} u_{1}^{j} d d^{c} u_{2}^{j} \wedge \ldots \wedge d d^{c} u_{q}^{j} \wedge T \wedge d d^{c} \phi+
\end{aligned}
$$

sum of integrals involving $d d^{c}(\phi h)$.

Recall that if positive measures $\mu_{k} \leftrightarrow \mu$ weakly on an open $\Omega$ set then $\widetilde{\lim }_{k \mapsto \infty} \mu_{k}(K) \leqq \mu(K)$ for each compact $K \subset \Omega$ and $\mu(V) \leqq \underline{\lim } \mu_{k}(V)$ for each open set. Hence, if $V$ is an open set such that $\mu_{k}(\partial V)=\mu(\partial V)=0$ for all $k$, then $\mu(V)=\lim _{k} \mu_{k}(V)$. We can assume that all the measures, which are coefficients of the currents we work with, have no mass on $\partial B$.

On support of $d d^{c}(\phi h), u_{l}^{\prime}=A \psi$, so we have only $q-1$ nonconstant sequences $\left(u_{m}^{\prime}\right)$ so for every integral involving $d d^{c}(\phi h)$ we have convergence, but as it is shown from the above development the limit of the sum of integrals involving $d d^{c}(\phi h)$ is zero, (observe that the manipulations are purely algebraic) so

$$
\int u_{1} d d^{c} u_{2} \wedge \ldots \wedge T^{\prime} \wedge d d^{c} \phi \leqq \underline{\lim }_{j} \int u_{1}^{j} d d^{c} u_{2}^{\prime} \wedge \ldots \wedge d d^{c} u_{q}^{j} \wedge T^{\prime} \wedge d d^{c} \phi .
$$

Now assume $x \in M\left(u_{1}\right) \cap \ldots \cap M\left(u_{q}\right) \cap \operatorname{supp} T:=M$. Since this set is in the envelope of $p-q+1$ pseudoconvexity of the complement we can construct finitely many Hartogs figures to absorb successively all of $M$. Given a Hartogs 
figure $H$ and $x \in \hat{H} \cap M$, we can construct a smoothly bounded neighborhood $\omega \Subset \hat{H}$ of $x$ and smooth functions $\left(\phi_{i}\right)_{i \leq s}$ vanishing in a neighborhood of $\partial \omega \cap M$ such that $d d^{c} \phi_{1} \wedge \gamma_{1} \geqq 0$ and $\sum_{i}^{s} d d^{c} \phi_{1} \wedge \gamma_{1}>0$ in a neighborhood of $x$, where the $\gamma_{1}^{\prime} s$ are closed forms of bidegree $(p-q, p-q)$. We compute the integrals in the same way as above, where now $T^{\prime}$ denotes $T \wedge \gamma_{1}$. Since the support of $\phi h$ is contained in the set where we have already proved convergence, the sum of integrals involving $d d^{c}(\phi h)$ is going to converge to zero as $j \mapsto \infty$, and then we add up with respect to $1 \leqq i \leqq s$. Hence,

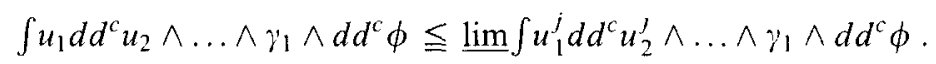

This finishes the convergence proof for general $q$. The last part follows immediately.

Corollary 3.6 Let $\Omega, T, u_{1}, \ldots, u_{q}$ and $u_{j} \geqq u_{k}$ be as in theorem. Assume that $A^{2(p-m+1)}\left(M\left(u_{j_{1}}\right) \cap \ldots \cap M\left(u_{J_{m}}\right) \cap \operatorname{supp} T\right)=0$ for all $j_{1}, \ldots, j_{m} \leqq q$. Then $u_{1} d d^{c} u_{2} \wedge \ldots \wedge d d^{c} u_{q} \wedge T$ has locally bounded mass and $u_{1}^{\prime} d d^{c} u_{2}^{\prime} \wedge \ldots \wedge d d^{c} u_{q}^{\prime}$ $\wedge T \mapsto u_{1} d d^{c} u_{2} \wedge \ldots \wedge d d^{c} u_{q} \wedge T$ in $\Omega$.

Proof. The assumption on the Hausdorff dimension of $X:=M\left(u_{j_{1}}\right) \cap \ldots$ i $M\left(u_{l m}\right) \cap \operatorname{supp} T$, implies that $X$ is in the envelope of $p-m+1$ pseudoconvexity of $\Omega \backslash X$, as in the proof of Corollary 3.4 so the result follows from Theorem 3.5.

A weaker form of the corollary was proved recently by Demailly [De]. He requires that $A^{2 p-2 m+1}\left(M\left(u_{f_{1}}\right) \cap \ldots \cap M\left(u_{\jmath_{m}}\right) \cap \operatorname{supp} T\right)=0$. The case when $M\left(u_{j}\right)$ is empty, $1 \leqq j \leqq q$ is due to Bedford and Taylor [BT].

Remark 3.7 Theorem 3.5 gives a Thullen type extension for currents of type $u T$. More precisely, let $T$ be a positive closed current in $\Omega$, of bidimension $(p, p)$. Let $u$ be a plurisubharmonic function in $\Omega$ and $V$ an analytic subvariety of dimension $p$ in $\Omega$. If $M(T, u)$ is contained in $V$ and $\Omega \backslash M(T, u)$ intersects every irreducible branch of $V$ then $M(T, u)=\varnothing$. The example of ShiffmanTaylor in [Siu] is a case where $V$ is of codimension 1 in $\mathbb{C}^{2}$ and $u d d^{c} u$ has locally bounded mass in $\mathbb{C}^{2} \backslash V$ and $M(T, u)=V$.

A natural question about the operator $\left(d d^{c}\right)^{k}$ is the following: Let $\left(u_{l}\right)$ be a sequence of plurisubharmonic functions in $\Omega$. Find the right notion of convergence such that $u_{j} \mapsto u$ implies $\left(d d^{c} u_{j}\right)^{k} \mapsto\left(d d^{c} u\right)^{k}$ in the sense of currents. Cegrell [Ce] and Lelong [Le] observed that the convergence of $\left(u_{j}\right)$ to $u$ in $L_{\mathrm{loc}}^{p}, p<\infty$, is not enough. We start with a refinement of their example.

Proposition 3.8 There exists a uniformly bounded sequence $\left(v_{p}\right)$ of plurisubharmonic functions in the unit bidisc $\Delta \subset \mathbb{C}^{2}$ such that:

(i) $v_{p}(z) \mapsto v(z)$ for every $z \in \Delta$ except on a set of Hausdorff dimension 2. More precisely, given any $\varepsilon>0$ and $\alpha>0$, there are balls $B\left(x_{t}, r_{i}\right)$ with $\sum r_{i}^{2+\varepsilon}<\infty$ such that for $p>p(\varepsilon), v_{p}>v-\varepsilon$ on $B \backslash \bigcup B\left(x_{i}, r_{i}\right)$.

(ii) $\left(d d^{c} v_{p}\right)^{2}$ does not converge to $\left(d d^{c} v\right)^{2}$. 
Proof. Let $v_{p}(z, w):=\left|z^{2 p}+w^{2 p}\right|^{1 /(2 p)}$ and $v(z, w)=\max (|z|,|w|)$. Clearly $0 \leqq$ $v_{p} \leqq 2^{1 / p} v$. Let $\omega:=\{|z| \neq|w|\}$. We have uniform convergence on compact subsets of $\omega$. Since through every point $q$ in $\mathbb{C}^{2}$ there exists a disc $\Delta_{q}$ on which $v_{p}$ is harmonic, it follows that $\left(d d^{c} v_{p}\right)^{2}=0$, see [FS1, Lemma 6.9]. On the other hand one computes easily that $\left(d d^{c} v\right)^{2} \neq 0$ since $v=1$ on the boundary of the unit polydisc but we do not have that $1 \leqq v$ as should be the case by maximum principle if $\left(d d^{c} v\right)^{2}=0$, see [BT].

We now study for which points $w=z e^{i \theta}, v_{p}(z, w)$ does not converge to $v(z, w)$. We have $v_{p}\left(z, e^{i \theta} z\right)=|z|\left|1+e^{2 i p \theta}\right|^{1 /(2 p)}=2^{1 / 2 p}|z||\cos p \theta|^{1 /(2 p)}$.

Let $E=\left\{e^{l \theta} ; \lim \inf _{p}|\cos p \theta|^{1 / p}<1\right\}$. Let $\varepsilon_{k}=1-1 / k$. Then $E \subset$ $\bigcup_{k} \cap_{N} \bigcup_{p>N}\left\{|\cos p \theta|<\varepsilon_{k}^{p}\right\}=: \bigcup E_{k}$. The set $\left\{|\cos p \theta|<\varepsilon_{k}^{p}\right\}$ is contained in a union of $2 p$ intervals of length at most $3 \varepsilon_{k}^{p} / p$ for $p \geqq p(k)$. Given $\alpha>0$ we have $\Lambda^{x}(E) \leqq \sum_{k} \Lambda^{x}\left(E_{k}\right)$, and $\Lambda^{\alpha}\left(E_{k}\right) \leqq c \sum_{p>N} p\left(\varepsilon_{k}^{p}\right)^{\alpha}=o(1)$ as $N \mapsto \infty$ so for every $k, \Lambda^{x}\left(E_{k}\right)=0$. Hence $A^{\alpha}(E)=0$. It follows easily that $v_{p}(z, w) \mapsto v(z, w)$ except on a set of Hausdorff dimension 2. Indeed, given any $\varepsilon>0$ and $\alpha>0$, there are balls $B\left(x_{i}, r_{l}\right)$ with $\sum r_{l}^{2+x}<\varepsilon$ and such that for $p>p(\varepsilon), v_{p} \geqq v-\varepsilon$ on $B \backslash \bigcup_{i}\left(B\left(x_{i}, r_{l}\right)\right.$.

The previous example is quite sharp as the following result* shows.

Theorem 3.9 Let $T_{j}$ be a sequence of positive closed currents of bidimension $(p, p)$ in an open set $\Omega$ in $\mathbb{C}^{n}$. Let $A$ be a subset in $\Omega$ with $\Lambda^{2 p}(A)=0$. Let $\left(u_{j}\right)$ be a bounded sequence of plurisubharmonic functions and $u$ a plurisubharmonic function in $\Omega$, whose restriction to $\Omega \backslash A$ is continuous. Assume

(i) $T_{j} \mapsto T$ as currents.

(ii) $u_{j} \mapsto u$ in $L_{\text {loc }}^{\mathrm{l}}$.

(iii) For every compact $K \subset \Omega \backslash A$ and $\varepsilon>0$ there exists $j(K)$ such that for $j \geqq j(K) u_{j} \geqq u-\varepsilon$.

Then $u_{j} T_{j} \mapsto u T$ and $d d^{c} u_{j} \wedge T_{j} \mapsto d d^{c} u \wedge T$.

Proof. Observe that we do not assume that $A$ is closed.

Let $S$ be a limit point of the bounded sequence of currents $u_{j} T_{j}$. By Proposition 3.2 we know that $S \leqq u T$. Let $\gamma$ be a positive form with compact support in $\omega \Subset \omega_{1} \Subset \Omega$. Since $\left(u_{j}\right)$ is bounded, we can assume that $0 \leqq u_{j} \leqq M$. We have to show that $\int u T \wedge \gamma \leqq \lim \inf \int u_{j} T_{j} \wedge \gamma$.

Since the mass of $\left(T_{j}\right)$ is bounded by a constant $C$ on $\omega_{1}$, for every ball $B(x, r) \subset \omega_{1}$, we have [Lel]

$$
\sigma_{T} B(x, r) \leqq C r^{2 p}
$$

Fix $\varepsilon>0$ and let $B\left(x_{j}, r_{j}\right)$ be a sequence of balls such that $A \subset \bigcup B\left(x_{i}, r_{i}\right)$ and $\sum_{i} r_{i}^{2 p}<\varepsilon$. It follows from (1) that there exists an open set $U \supset A \cap \omega$ such that $\sigma_{T_{j}}(U)<\varepsilon$ independently of $j$. Let $K=\operatorname{supp} \gamma \cap(\Omega \backslash U)$. Since $u_{j} T_{j} \geqq 0$,

${ }^{*}$ Note. We thank Russakovski for pointing out that a weaker version of Theorem 3.9 has been proved by Ronkin [Ro]. 
we have for $j \geqq j(K)$

$$
\begin{aligned}
\int u_{j} T_{j} \wedge \gamma & =\int_{(1) \backslash U}\left(u_{j}-u\right) T_{j} \wedge \gamma+\int_{(\omega \backslash U} u T, \wedge \gamma+\int_{U} u_{j} T, \wedge \gamma \\
& \geqq-C \varepsilon+\int_{\omega \backslash U} u T_{j} \wedge \gamma .
\end{aligned}
$$

Let $\tilde{u}$ be a continuous extension of $u$ from $\operatorname{supp} \gamma \backslash U$ to $\operatorname{supp} \gamma, 0 \leqq \tilde{u} \leqq M$. Since for all $j, \sigma_{T j}(U)<\varepsilon$, the last integral is close to $\int_{(1)} \tilde{u} T, \wedge \gamma$ which converges to $\int u T \wedge \gamma$. We have then proved that

$$
\int u T \wedge \gamma \leqq \lim _{j} \int u_{j} T_{j} \wedge \gamma
$$

which is what we wanted to show.

\section{Bezout theorem for currents in $\mathbb{P}^{k}$}

Let $\omega$ be the standard Kahler form on $\mathbb{P}^{k}$, normalized such that $\int_{\mathbb{P}^{k}} \omega^{k}=1$. If $\pi: \mathbb{C}^{k+1} \mapsto \mathbb{P}^{k}$ is the canonical projection, then $\pi^{*} \omega=d d^{c} \log \|z\|$ where $z=\left(z_{0}, z_{1}, \ldots, z_{k}\right)$ and \|\| is the Euclidean norm in $\mathbb{C}^{k+1}$. Given a pusitive closed current $T$ of bidimension $(p, p)$ we define $\|T\|:=\int T \wedge \omega^{p}$. When $T$ corresponds to integration on an analytic manifold $V$, then $\|T\|$ is just the $2 p$ volume of $V$. We will need the following standard result.

Proposition 4.1 Let $R$ be a closed positive $(1,1)$ current on $\mathbb{P}^{k}$.

(i) There exists a plurisubharmonic function $v$ on $\mathbb{C}^{k+1}$ such that $\pi^{*} R=$ $d d^{c} v, v(\lambda z)=c \log |\lambda|+v(z)$ for $\lambda \in \mathbb{C}, z \in \mathbb{C}^{k}$.

(ii) If $v$ is a plurisubharmonic function on $\mathbb{C}^{k+1}$, satisfying the previous homogeneity condition, then there is a decreasing sequence $\left(v_{s}\right)$ of plurisubharmonic functions on $\mathbb{C}^{k+1}$ satisfying the homogeneity condition, such that $v_{\varepsilon} \in C^{\infty}\left(\mathbb{C}^{k+1} \backslash\{0\}\right)$ and $v_{\varepsilon} \searrow v$.

Proof. A proof of (i) can be found in Theorem 4.1 of [FS1], see also [LG].

For simplicity assume $c=1$. If $\alpha$ is an approximation of the identity in $\mathbb{C}^{k+1}$ depending only on $\|z\|$, we define

$$
v_{\varepsilon}(z):=\frac{1}{\|z\|^{2(k+1)}} \int v(w) \alpha_{\varepsilon}\left(\frac{z-w}{\|z\|}\right) d \lambda(w)=\int v(z-\|z\| w) \alpha_{\varepsilon}(w) d \lambda(w)
$$

where $\lambda$ denotes Lebesgue measure. The proof is then the same as in Theorem 7.6 of Lelong and Gruman [LG].

Proposition 4.2 Let $R$ be a positive closed current of bidegree $(1,1)$ on $\mathbb{P}^{k}$. Let $v$ be a plurisubharmonic function on $\mathbb{C}^{k+1}$ satisfying:

(i) $\pi^{*} R=d d^{c} v$

(ii) $v(\lambda z)=c \log |\lambda|+v(z), \lambda \in \mathbb{C}$.

Then $\|R\|=c$. 
Proof. We can assume $c=1$. Let $\left(v_{\varepsilon}\right)$ be the sequence of plurisubharmonic functions constructed in Proposition 4.1. Consider the positive closed current on $\mathbb{P}^{k},\left(R_{\varepsilon}\right)$, defined by the relation $\pi^{*} R_{\varepsilon}=d d^{c} v_{\varepsilon}$. Let $u_{\varepsilon}\left[z_{0}: \ldots: z_{k}\right]=$ $v_{\varepsilon}\left(z_{0}, \ldots, z_{k}\right)-\log \|z\|$. The function $u_{\varepsilon}$ is smooth and well defined on $\mathbb{P}^{k}$. Moreover $R_{\varepsilon}-\omega=d d^{c} u_{\varepsilon}$. Since $v_{\varepsilon} \mapsto v$ in $L_{\text {loc }}^{1}$, then $R_{\varepsilon} \mapsto R$ in the sense of currents. Using Stoke's theorem we get:

$$
\begin{aligned}
\|R\| & =\int R \wedge \omega^{k-1}=\lim _{\varepsilon \rightarrow 0} \int R_{\varepsilon} \wedge \omega^{k-1} \\
& =\lim _{\varepsilon \mapsto 0} \int \omega^{k}+d d^{c} u_{\varepsilon} \wedge \omega^{k-1}=\int \omega^{k}=1 .
\end{aligned}
$$

Remark 4.3 Let $P$ be a homogeneous polynomial of degree $d$ in $\mathbb{C}^{k+1}$. Let $V=\left\{z \in \mathbb{P}^{k}, P(z)=0\right\}$ and $[V]$ the current of integration on $V$. Since $\pi^{*}[V]=$ $d d^{c} \log |P|$, we get $\operatorname{vol}(V)=\|V\|=\int[V] \wedge \omega^{k-1}=d$.

Let $T$ be a positive closed current of bidimension $(p, p)$ in $\mathbb{P}^{k}$. Let $R_{1}, \ldots, R_{q}$ be closed positive currents of bidegree $(1,1)$. Locally in $\mathbb{P}^{k}$ each $R_{f}$ can be written as $d d^{c} u_{j}$ where $u_{j}$ is a plurisubharmonic function. We will say that $\left(T, R_{1}, \ldots, R_{q}\right)$ admit a wedge product if for every $x \in \mathbb{P}^{k}$, there exists an open set $\omega$ containing $x$, and plurisubharmonic functions $\left(u_{1}, \ldots, u_{q}\right)$ on $\omega$ such that for every $j, 1 \leqq j \leqq q, R_{j}=d d^{c} u_{j}$, and moreover for all $i_{1}, \ldots, i_{m} \in[1, q]$, the set

$$
X:=\operatorname{Supp} T \cap M\left(u_{i_{1}}\right) \cap \ldots \cap M\left(u_{l_{m}}\right) \cap \omega
$$

is in the envelope of $p-m+1$ pseudoconvexity of $\omega \backslash X$.

Under this assumption and using Theorem 3.5 we can define the wedge product $T \wedge R_{1} \wedge \ldots \wedge R_{q}$.

Theorem 4.4 Let $T$ be a positive closed current of bidimension $(p, p)$ on $\mathbb{P}^{k}$. Let $R_{1}, \ldots, R_{q}$ be positive closed currents of bidegree $(1,1)$ on $\mathbb{P}^{k}$. Assume that $\left(T, R_{1}, \ldots, R_{q}\right)$ admit a wedge product. Then

$$
\left\|T \wedge R_{1} \ldots \wedge R_{q}\right\|=\|T\|\left\|R_{1}\right\| \ldots\left\|R_{q}\right\| .
$$

In particular $T \wedge R_{1} \wedge \ldots \wedge R_{q}$ is nonzero and $\operatorname{supp} T \cap \operatorname{supp}\left(R_{1}\right) \cap \ldots \cap$ $\operatorname{supp}\left(R_{q}\right) \neq \varnothing$.

Proof. Without loss of generality we can assume that $\|T\|=\left\|R_{1}\right\|=\ldots=$ $\left\|R_{q}\right\|=1$. Assume $\pi^{*} R_{q}=d d^{c} v, v(\lambda z)=\log \left|\lambda_{1}\right|+v(z)$. Then as in the proof of Proposition 4.2, $R_{q}=\lim _{\varepsilon \mapsto 0} R_{q}^{\varepsilon}$ where $\pi^{*} R_{q}^{\varepsilon}=d d^{c} v_{\varepsilon}$. The assumption that $\left(T, R_{1}, \ldots, R_{q}\right)$ admit a wedge product and Theorem 3.5 imply that $T \wedge R_{1} \wedge$ $\ldots \wedge R_{q}=\lim _{\varepsilon \rightarrow 0} T \wedge R_{1} \wedge \ldots \wedge R_{q-1} \wedge R_{q}^{\varepsilon}$. (Indeed convergence of currents has to be checked locally, and $v_{\varepsilon} \searrow v$ in a given chart in $\mathbb{P}^{k}$.)

We can also assume that $p+q=k$. Hence using Stokes theorem we get if $u_{\varepsilon}[z]=v_{\varepsilon}(z)-\log |z|$

$$
\begin{aligned}
\int T \wedge R_{1} \wedge \ldots \wedge R_{q} & =\lim _{\varepsilon \rightarrow 0} \int T \wedge R_{1} \wedge \ldots \wedge R_{q-1} \wedge R_{q}^{\varepsilon} \\
& =\lim _{\varepsilon \mapsto 0} \int T \wedge R_{1} \wedge \ldots \wedge R_{q-1} \wedge\left(\omega+d d^{c} u_{\varepsilon}\right) \\
& =\int T \wedge R_{1} \wedge \ldots \wedge R_{q-1} \wedge \omega .
\end{aligned}
$$


Repeating the procedure we then show that

$$
\int T \wedge R_{1} \wedge \ldots \wedge R_{q}=\int T \wedge \omega^{q}=1 .
$$

Remark 4.5 Let $P_{1}, \ldots, P_{q}$ be homogeneous polynomials of degree $d_{1}, \ldots, d_{q}$ in $\mathbb{C}^{k+1}$. Let $V_{j}=\left\{z \in \mathbb{P}^{k} ; P_{j}(z)=0\right\}$. Assume $\operatorname{codim}\left(V_{\lambda_{1}} \cap \ldots \cap V_{J_{m}}\right)=m$. Then the currents $\left(\left[V_{1}\right], \ldots,\left[V_{q}\right]\right)$ admit a wedge product. Indeed if $u$, is such that on a given chart on $\mathbb{P}^{k}, d d^{c} u_{j}=\left[V_{j}\right]$, then $M\left(u_{i_{1}}\right) \cap \ldots \cap M\left(u_{t_{m}}\right)$ has dimension $k-m$, so is in the $k-m+1$ envelope of the complement. So Theorem 4.4 and Remark 4.3 gives in this case Bezout's theorem. See Demailly [De] who shows that $\left[V_{1}\right] \wedge \ldots \wedge\left[V_{q}\right]$ is the current of integration of the intersection with multiplicity.

Theorem 4.6 Let $T$ be a nonzero, positive closed current of bidegree $(p, p)$ in $P^{k}$. Let $X=\operatorname{supp} T$. For any algebraic variety $V=\left\{h_{1}=\ldots=h_{l}=0\right\}, X \cap$ $V \neq \varnothing$ provided $p+l \leqq k$ and provided $V$ is a geometric complete intersection.

Proof. Let $\omega$ be the Kahler form on $\mathbb{P}^{k}$. Replacing $T$ by $T \wedge \omega^{k-(p+l)}$ we can assume that $p+l=k$. We assume that $X \cap V=\varnothing$.

Define

$$
u\left[z_{0}: \ldots: z_{k}\right]=\log \frac{\|z\|^{2 s}}{\left|h_{1}\right|^{2}+\ldots+\left|h_{l}\right|^{2}}
$$

where we have assumed that the polynomials $h_{j}$ are homogenous of degree $s$.

The function $u$ is smooth in $\mathbb{P}^{k} \backslash V$. Let $S$ be the current in $\mathbb{P}^{k}$ defined by the relation:

$$
\pi^{*} S=\left(d d^{c} \log \left(\left|h_{1}\right|^{2}+\ldots\left|h_{l}\right|^{2}\right)\right)^{l-1} .
$$

We show at first that in $\mathbb{P}^{k} \backslash V$, we have

$$
d d^{c} u \wedge S=2 s \omega \wedge S \text {. }
$$

In local coordinates

$$
d d^{c} u \wedge S=d d^{c} \log \|z\|^{2 s} \wedge S-\left(d d^{c} \log \|h\|^{2}\right)^{l}
$$

and the last term is zero outside $V$. Indeed, if for example $h_{1} \neq 0, \log \|h\|^{2}=$ $\log \left|h_{1}\right|^{2}+\log \left(1+\frac{\left|h_{2}\right|^{2}}{\left|h_{1}\right|^{2}}+\ldots+\frac{\left|h_{1}\right|^{2}}{\left|h_{1}\right|^{2}}\right)$. If we consider the variety $\frac{h_{m}}{h_{1}}=c^{t e}$, we see that $d d^{c} \log \|h\|^{2}$ has at least $k-l+1$ zero eigenvalues, the result follows.

Let $\theta$ be a nonnegative test function with support in $\mathbb{P}^{k} \backslash V$ and with value 1 in a neighborhood of $X$. Since $S$ is smooth and closed in a neighborhood of $\operatorname{supp} T$, the current $T \wedge S$ is well defined and

$$
\left\langle T \wedge S, \theta d d^{c} u\right\rangle=\left\langle T \wedge S, d d^{c}(u \theta)\right\rangle=\left\langle d d^{c} T \wedge S, u \theta\right\rangle=0
$$

and hence $T \wedge S \wedge \omega=0$. We can vary slightly the $h_{j}^{\prime}$ s to obtain varieties $V$, close to $V$ and still not intersecting $X$. We obtain for the corresponding forms 
$S_{l}$, that $T \wedge S_{i} \wedge \omega=0$. It is clear that if we take enough perturbations of $V$, this implies that $T=0$, a contradiction.

Theorem 4.7 Let $T$ be a positive, closed $(1,1)$ current on $\mathbb{P}^{k}$. Assume $\pi^{*} T=$ $d d^{c} v$ with $v$ plurisubharmonic and continuous on $\mathbb{C}^{k+1} \backslash\{0\}$. Let $X_{l}=\operatorname{supp} T^{l}$. Then for $2 l \leqq k, X_{l}$ is connected.

Proof. We first observe that a positive closed current $S$ of bidegree $(l, l)$ is a limit of smooth positive closed currents. Indeed it is enough to take an approximation of identity in $\operatorname{Aut}\left(\mathbb{P}^{k}\right)$ and average the current $S$ with respect to the approximation of identity. Let $\rho_{\varepsilon}(g)$ be an approximation of identity on the group $U(k)$ which acts transitively on $\mathbb{P}^{k}$. Let $\mu$ be the Haar measure for $U(k)$. For a positive $(l, l)$ current $S$ define $S_{\varepsilon}$ by

$$
S_{\varepsilon}=\int_{U(k)} \rho_{\varepsilon}(g)\left(g_{*} S\right) d \mu(g) .
$$

If $\rho_{\varepsilon} \geqq 0, S_{\varepsilon}$ is positive, closed and smooth [deRh]. The previous formula has to be understood as follows: If $\phi$ is a test form,

$$
\left\langle S_{\varepsilon}, \phi\right\rangle=\int \rho_{\varepsilon}(g)\left\langle S, g^{*} \phi\right\rangle d \mu(g) .
$$

It is also known, Dold [Do] or de Rham [deRh], that there exists a constant $c$ such that $S-c \omega^{l}=d d^{c} H$ where $H$ has integrable coefficients. Observe that since $S \geqq 0$ then $c \geqq 0$.

Since $T$ has locally a continuous potential $u$, i.e. $d d^{c} u=T$, we can define $T^{l}$ for any $l \leqq k$. Assume there exists two disjoint open sets $U_{1}, U_{2}$ each one intersecting $X_{l}=\operatorname{supp} T^{l}$ and such that $X_{l} \cap \partial U_{1}=X_{l} \cap \partial U_{2}=\varnothing$. Let $S_{1}=$ $T^{l} \mid U_{1}$ and $S_{2}=T^{l} \mid U_{2}$. Clearly $S_{1}$ is a positive closed current. Let $\left(S_{1}^{n}\right)$ be a sequence of smooth positive closed currents $S_{1}^{n} \mapsto S_{1}$. Let $B$ be a ball where $T=d d^{c} u$. Since all the currents are positive, hence with measure coefficients, we have $u S_{1}^{n} \mapsto u S_{1}$, on $B$ hence $d d^{c} u \wedge S_{1}^{n} \mapsto d d^{c} u \wedge S_{1}$. Inductively we get $\left(d d^{c} u\right)^{l} \wedge S_{1}^{n} \mapsto\left(d d^{c} u\right)^{l} \wedge S_{1}$, hence $T^{l} \wedge S_{1}^{n} \mapsto T^{l} \wedge S_{1}$. Since $T^{l}$ has no mass on $\partial U_{2}$ we also have $S_{2} \wedge S_{1}^{n} \mapsto S_{2} \wedge S_{1}$.

Let $s=k-2 l$ and let $S_{2}=c_{2} \omega^{l}+d d^{c} H_{2}$. We have

$$
\begin{aligned}
\int S_{2} \wedge S_{1} \wedge \omega^{s} & =\lim _{n} \int S_{2} \wedge S_{1}^{n} \wedge \omega^{s} \\
& =\lim _{n} \int c_{2} S_{1}^{n} \wedge \omega^{l+s}+\int d d^{c} H_{2} \wedge S_{1}^{n} \wedge \omega^{s} .
\end{aligned}
$$

Since $S_{1}^{n}$ are smooth, the last integral is zero. Hence

$$
\int S_{2} \wedge S_{1} \wedge \omega^{s}=c_{2} \int \omega^{l+s} \wedge S_{1}
$$

But $S_{2} \neq 0$ implies $c_{2} \neq 0$ and $S_{1} \neq 0$ implies that $S_{1} \wedge \omega^{l+s} \neq 0$. Consequently the previous relation shows that $S_{1} \wedge S_{2} \neq 0$, contradicting the assumption that $\operatorname{supp} S_{1} \cap \operatorname{supp} S_{2}=\varnothing$. 


\section{Examples from holomorphic dynamics in $\mathbb{P}^{k}$}

For background on this section we refer to [FS1], [FS2] and [HP].

Let $f$ be a holomorphic surjective map on $\mathbb{P}^{k}$. Let $F=\left[F_{0}: \ldots: F_{k}\right]$ be a lifting of $f$ to $\mathbb{C}^{k+1}$. Let $d$ be the common degree of the polynomials $F_{j}$. The fact that $f$ is holomorphic implies that $F^{-1}(0)=(0)$ and hence, there exists a constant $c>0$ such that $1 / c\|z\|^{d} \leqq\|F(z)\| \leqq c\|z\|^{d}$. Hence out of 0 we have

$$
\left|1 / d^{n+1} \log \left\|F^{n+1}\right\|-1 / d^{n} \log \left\|F^{n}\right\|\right| \leqq c_{1} / d^{n}
$$

and the function

$$
G(z):=\lim _{n \rightarrow \infty} 1 / d^{n} \log \left\|F^{n}(z)\right\|
$$

is continuous on $\mathbb{C}^{k+1} \backslash\{0\}$ since the convergence is uniform. Clearly we have:

(i) $G(\lambda z)=\log |\lambda|+G(z), \lambda \in \mathbb{C}$

(ii) $G$ is plurisubharmonic in $\mathbb{C}^{k+1}$

(iii) $G((F(z))=d G(z)$.

Definition 5.1 Let $f: \mathbb{P}^{k} \mapsto \mathbb{P}^{k}$ be in the space $H_{d}$ of holomorphic self maps on $\mathbb{P}^{k}$ given by polynomials of degree $d$. For $0 \leqq l \leqq k-1$, a point $p \in \mathbb{P}^{k}$ belongs to the Fatou set $F_{l}$ if there exists a neighborhood $U(p)$ such that for every $q \in U(p)$ there exists a complex variety $X_{q}$ of codimension $l$ such that $\left\{f^{n} \mid X_{q}\right\}$ is equicontinuous. Observe that $F_{0}$ is the largest open set where $f^{n}$ is equicontinuous. We call $F_{0}$ the Fatou set. We have $F_{0} \subset F_{1} \subset \ldots \subset F_{h-1}$.

Let $J_{l}:=\mathbb{P}^{k} \backslash F_{l}$, we call $J_{l}$ the Julia set of order $l$.

Let $T$ be the $(1,1)$ positive closed current defined on $\mathbb{P}^{k}$ by the relation $\pi^{*} T=$ $d d^{c} G$. Since $G$ is continuous on $\mathbb{C}^{k+1} \backslash\{0\}, T$ has a continuous potential on any chart in $\mathbb{P}^{k}$, for example if $z_{0} \neq 0, T=d d^{c} G\left(1, z_{1}, \ldots, z_{k}\right)$. Hence we can define the closed positive currents of bidegree $(l, l)$ by the relation $\pi^{*}\left(T^{l}\right)=$ $\left(d d^{c} G\right)^{l}$ for any $1 \leqq l \leqq k$. We define $J_{l-1}^{\prime}=\operatorname{supp} T^{l}, 1 \leqq l \leqq k$, and $F_{l-1}^{\prime}=$ $\mathbb{P}^{k} \backslash J_{l-1}^{\prime}$.

Theorem 5.2 Let $f: \mathbb{P}^{k} \mapsto \mathbb{P}^{k}$ be a surjective holomorphic map of degree $d \geqq 2$ on $\mathbb{P}^{k}$. Let $T$ be the positive closed $(1,1)$ current associated to $f$. Then

(i) $J_{k-1}^{\prime}$ is nonempty and for every $0 \leqq l<k, f\left(J_{l}^{\prime}\right)=f^{-1}\left(J_{l}^{\prime}\right)=J_{l}^{\prime}$. Moreover $J_{l}^{\prime} \subset J_{l}$ for $0 \leqq l<k$.

(ii) Support $T=J_{0}^{\prime}=J_{0}$ and $F_{0}$ is a domain of holomorphy. For $0 \leqq l<$ $k, F_{l}^{\prime}$ is $(k-l-1)$ pseudoconvex.

(iii) $J_{l-1}^{\prime}$ is connected if $2 l \leqq k$.

(iv) If $X$ is an algebraic variety of dimension $r$, which is a complete intersection, then $T^{r} \wedge[X]$ is a positive measure of total mass vol( $\left.X\right)$. Moreover $X$ intersects every component of supp $T^{r}$.

Proof. It follows from Proposition 4.2 that $\|T\|=1$ since $G(\lambda z)=\log |\lambda|+$ $G(z)$. Theorem 4.4 implies that for every $1 \leqq l \leqq k\left\|T^{\prime}\right\|=1$, in particular $T^{k}$ is a probability measure, hence $J_{k-1}^{\prime}=\operatorname{supp} T^{k}$ is nonempty. 
Let $G_{n}:=1 / d^{n} \log \left\|F^{n}\right\|$ and observe that $G_{n}$ are smooth and converge uniformly to $G$ on $\mathbb{C}^{k+1} \backslash\{0\}$. If $\left(d d^{c} G_{n}\right)^{l} \mapsto 0$, then $\left(d d^{c} G_{n} \circ F\right)^{l}=$ $\left(d d^{c}\left(G_{n+1} d\right)\right)^{l} \mapsto 0$, hence $F_{l}^{\prime}$ and $J_{l}^{\prime}$ are totally invariant.

We prove now that $J_{l}^{\prime} \subset J_{l}, 0 \leqq l<k$. We have to show that if $p \in F_{l}$ then $T^{l+1}$ vanishes in a neighborhood of $p$. Let $U$ be a neighborhood of $p, q \in U$, and let $X_{q}$ be an analytic variety of codimension $l$ through $q$ such that $f^{n} \mid X_{q}$ is equicontinuous. Let $f^{n_{1}} \mid X_{q} \mapsto h$. This means that we can find non zero holomorphic functions $\lambda_{n_{i}}$ on $X_{q}$ such that $\frac{F^{n_{i}}}{\hat{\lambda}_{n_{l}}}$ converge to a holomorphic function $H$. We can write

$$
G_{n_{t}}=1 / d^{n_{t}} \log \| F^{n_{t}} / \lambda_{n_{t}}\left|+1 / d^{n_{t}} \log \right| \lambda_{n_{t}} \mid
$$

The first sequence converges uniformly to zero on $\pi^{-1}\left(X_{q}\right)$ and the sequence $1 / d^{n} \log \left|\lambda_{n_{1}}\right|$ is harmonic on any analytic disc in $\pi^{-1}\left(X_{q}\right)$. So $G$ is harmonic on any analytic disc in $\pi^{-1}\left(X_{q}\right)$. Hence if $G_{1}$ is a local section of $G$ in $U, G_{1}$ is harmonic on any analytic disc contained in $X_{q}$. Assume that $U$ is a polydisc $\Delta^{k-l-1} \times \Delta^{l+1}$. For every $a \in \Delta^{k-l-1}$ and every $q$ in $a \times \Delta^{i+1}, X_{q}^{a}:=a \times \Delta^{l+1} \cap X_{q}$ is at least of dimension one, and $G_{1}$ is harmonic on any analytic disc contained in $X_{q}^{a}$. Therefore Lemma 6.9 in [FS2] implies that $\left(d d^{c} G_{1}(a, w)\right)^{l+1}=0$ in $a \times \Delta^{l+1}$. Since $a$ was arbitrary, standard results in slicing theory applied to the current $T^{l+1}$ [HS] or [Fe], imply that $T^{l+1}$ is zero on $U$.

(ii) We show that supp $T=J_{0}$. We have that supp $T \subset J_{0}$, in this case no slicing is needed since the first part of the proof shows that $G$ is pluriharmonic on $\pi^{-1}\left(F_{0}\right)$. Let $U$ be an open set disjoint from $\operatorname{supp} T$. We want to prove that $U \subset F_{0}$. We can assume $U$ is a ball. We know that $G$ is pluriharmonic on $\pi^{-1}(U)$, hence $G=\log |h|$ where $h$ is holomorphic on $\pi^{-1}(U)$. Since

$$
\left|G-G_{n}\right| \leqq c / d^{n}
$$

we get

$$
e^{-c} \leqq \frac{\left\|F^{n}\right\|}{\left|h^{d^{n}}\right|} \leqq e^{c}
$$

So the family $\left(f^{n}\right)$ is normal on $U$.

The fact that $F_{0}=\mathbb{P}^{k} \backslash J_{0}$ is pseudoconvex follows from Corollary 2.6. Since the Levi problem has a positive solution for domains in $\mathbb{P}^{k}$, it follows that $F_{0}$ is a domain of holomorphy. Corollary 2.6 also implies that $F_{l-1}^{\prime}$, the complement of $\operatorname{supp} T^{l}$ is $(k-l)$-pseudoconvex.

(iii) Theorem 4.7 shows that $J_{l-1}^{\prime}$ is connected if $l \leqq 2 k$.

(iv) Since $T$ has locally a continuous potential, $T^{r}$ and $[X]$ admit a wedge product. The current $[X]$ is given locally as $d d^{c} \log \left|P_{1}\right| \wedge \ldots \wedge d d^{c} \log \left|P_{k-r}\right|$ where $\left(P_{l}\right)$ are homogeneous polynomials. So we can apply Theorem 4.4 and we get that $\left\|T^{r} \wedge[X]\right\|=\|[X]\|$. Let $Y$ be a component of supp $T^{r}$. Assume $Y \cap X=\varnothing$. Let $U$ be a neighborhood of $Y$ such that $U \cap X=\varnothing$. Let $S=$ $T^{r} \mid U, S$ is a positive closed current of bidegree $(r, r)$ to which we can apply Theorem 4.6, hence supp $S$ intersects $X$, a contradiction. 
We next show that $f$ cannot be hyperbolic on $J_{l}^{\prime}$ for $l<k-1$. Let $f \in$ $H_{d}, f: \mathbb{P}^{k} \mapsto \mathbb{P}^{k}$. We define first hyperbolicity, see Ruelle [Ru]. Let $K \subset \mathbb{P}^{k}$ be a compact set. We assume that $K$ is surjectively forward invariant, that is $f(K)=K$. The space $\hat{K}=K^{\mathbb{N}}$ of orbits $\left\{x_{n}\right\}_{n=-\infty}^{0}, f\left(x_{n}\right)=x_{n+1}$ is compact in the product topology. By the tangentbundle $T_{k}$ of $\hat{K}$ we mean the space of $(x, \xi)$ where $x=\left\{x_{n}\right\} \in \hat{K}$ and $\xi \in T_{\mathbb{P}^{2}}\left(x_{0}\right)$ is a tangent vector. We give this tangentbundle the obvious topology. Then $f$ lifts to a homeomorphism $\hat{f}: \hat{K} \mapsto \hat{K}$ and $f^{\prime}$ lifts to a map $f^{\prime}$ on $T_{k}$ in the obvious way.

Definition 5.3 Let $K \subset \mathbb{P}^{k}$ be a compact surjectively forward invariant set. Then $f$ is hyperbolic on $K$ if there exists a continuous splitting $E^{u} \oplus E^{s}$ of the tangent space bundle of $\hat{K}$ such that $\hat{f}^{\prime}$ preserves the splitting and for some constants $C, c>0, \lambda>1, \mu<1$ depending on the choice of a Hermitian metric on $\mathrm{IP}^{2}$,

$$
\begin{aligned}
& \left|D \hat{f}^{n}(\xi)\right| \geqq c \lambda^{n}|\xi|, \xi \in E^{u} \\
& \left|D \hat{f}^{n}(\xi)\right| \leqq C \mu^{n}|\xi|, \xi \in E^{s}, \quad n=1,2, \ldots
\end{aligned}
$$

Theorem 5.4 Let $f: \mathbb{P}^{k} \mapsto \mathbb{P}^{k}, f \in H_{d}$. If supp $\mu=J_{k-1}^{\prime}$ is hyperbolic, then the unstable dimension of $J_{k-1}^{\prime}$ is equal to $k$.

Proof. Suppose the stable dimension is equal to $l \geqq 1$. Then through every point $x \in J_{k-1}^{\prime}$ there is an analytic disc $\Delta_{x}$ on which the family $\left(f^{n}\right)$ is equicontinuous. Hence if $G_{1}$ is a local potential for $\mu$, i.e. $\left(d d^{c} G_{1}\right)^{\hat{h}}=\mu$, then $G_{1}$ is harmonic on $\Delta_{x}$. The theorem will then be a consequence of the following lemma.

Lemma 5.5 Let $u$ be a continuous plurisubharmonic function in a ball $\bar{B} \subset \mathbb{C}^{k}$. Let $\left(d d^{c} u\right)^{k}=v$. Assume that through every point $x$ of $X:=\operatorname{supp} v$ there is an analytic disc $\Delta_{x}$ such that $u \mid \Delta_{x}$ is harmonic, then $v=0$.

Proof. We are going to prove that $u$ is maximal and hence $v=0$. Let $v$ be a continuous function on $\bar{B}$ which is plurisubharmonic on $B$, with $v \leqq u$ on $\partial B$. Let $M:=\sup _{B} v-u$, assume $M>0$. Let $K=\{z ; z \in B,(v-u)(z)=M\}$. Let $p$ be a peak point for a function $h \in C(K)$ which is a uniform limit of $K$ of holomorphic polynomials, i.e., $h(p)=1$ and $|h|<1$ on $K \backslash\{p\}$. If $p \in X$, then since $v \leqq u+M$ and $u \mid \Delta_{p}$ is harmonic then $v-u=M$ on $\Delta_{p}$, hence $\Delta_{p} \subset K$, contradicting that $p$ is a peak point for $h$. So $p \in B \backslash X$. Let $B(p, r) \Subset B$ be a ball such that $B(p, r) \cap X=\varnothing$. Since $\left(d d^{c} u\right)^{k}=0$ on $B(p, r)$, it follows from [GS] that given any $z \in B(p, r)$, there exists a probability measure $\sigma_{z}$ supported on $\partial B(p, r)$ such that $u(z)=\int u d \sigma_{z}$, and moreover for any continuous function on $\bar{B}(p, r)$, plurisubharmonic on $B(p, r)$

$$
\phi(z) \leqq \int \phi d \sigma_{z} .
$$

Since $M=v(p)-u(p) \leqq \int(u-v) d \sigma_{p} \leqq M$, we have that support $\sigma_{p}$ is contained in $K$, and $p$ cannot be a peak point for $h$, since $h(p)=\int h d \sigma_{p}$, hence $K$ is empty and $v=0$. 
Theorem 5.6 Let $f: \mathbb{P}^{k} \mapsto \mathbb{P}^{k}$ be a holomorphic map of degree $d \leqq 2$. Then $f$ cannot be hyperbolic on $\mathbb{P}^{k}$ nor on $J_{l}^{\prime}$ for $l<k-1$.

Proof. Assume $f$ is hyperbolic on $\mathbb{P}^{k}$. Since the critical set is nonempty the fibre dimension of $E^{u}$ is $\leqq k-1$. If $\operatorname{dim} E^{s}=k$ then all periodic orbits are attractive. Pick one, $p$, with immediate basin of attraction $\Omega$. Since $f$ is surjective, $\partial \Omega$ is a non empty, compact, forward invariant subset of $\mathbb{P}^{k}$. Hyperbolicity implies that orbits of points $q \in \Omega$ close to $\partial \Omega$ converge to $\partial \Omega$ contradicting that they are in the attractive basin of $p$. Hence $1 \leqq \operatorname{dim} E^{s} \leqq k-1$. Let $\operatorname{dim} E^{s}=l$. Then we have a foliation of $\mathbb{P}^{k}$ by stable manifolds of dimension $l$, and on each manifold the family $\left(f^{n}\right)$ is equicontinuous, so $\mathbb{P}^{k} \subset F_{k-l}$. Since $F_{k-1} \subset F_{k-1}$, we get $J_{k-1}=\varnothing$ which is a contradiction.

Let $l<k-1$ and assume $J_{l}^{\prime}$ is hyperbolic. Theorem 5.2(iv) shows that every component of $J_{l}^{\prime}$ intersects $C$, the critical set. Hence the fibre dimension of $E^{u} \leqq k-1$, so $\operatorname{dim} E^{s} \geqq l$. This implies that through every point $p$ in $J_{l}^{\prime} \supset \operatorname{supp} \mu$ there exists an analytic disc $\Delta_{p}$ on which $G_{1}$ is harmonic, $G_{1}$ is a local solution of $d d^{c} G_{1}=T$.

So Lemma 5.6 applies and $\mu=0$, a contradiction.

Acknowledgement. We thank G. Henkin and R. Narasimhan for attracting our attention to Oka's paper.

\section{References}

[AG] Andreotti, A., Grauert, H; Theoremes de finitude pour la cohomologie des espaces complexes. Bull. Soc. Math. Fr. 90 (1962), 193-259

[BT] Bedford, E., Taylor, B.A; A new capacity for plurisubharmonic functions. Acta Math. 149 (1982), 1-39

[Bi] Bishop, E; Conditions for analyticity of certain analytic sets. Mich. Math. J. 11 (1964), 289-304

[Ce] Cegrell, U; Discontinuite de l'operateur de Monge Ampere complexe, C.R.A.S. Paris, Serie I, Math. 296 (1983), 869-871

[CLN] Chern, S.S., Levine, H., Nirenberg, L; Intrinsic norms on a complex manifold. Global Analysis (Papers in Honour of K. Kodaira), Univ. Tokyo Press (1969)

[De] Demailly, J.P; Monge Ampere operators, Lelong numbers and intersection theory. Prepubl. Inst. Fourier 173

[de Rh] de Rham, G; Varietes differentiables. Paris, Hermann (1955)

[Do] Dold, A; Lectures on algebraic topology. Springer, Berlin Heidelberg New York (1972)

[Fe] Federer, H; Geometric Measure Theory. Springer, Berlin Heidelberg New York (1969)

[FS1] Fornæss, J.E., Sibony, N; Complex Dynamics in higher Dimension I. Asterisque (to appear)

[FS2] Fornæss, J.E., Sibony, N; Complex Dynamics in higher Dimension II. Preprint

[Fu] Fujita, O; Sur les familles d'ensembles analytiques. J. Math. Soc. Japan 1 (1964), $379-405$

[GS] Gamelin, T.W., Sibony, N; Subharmonicity for Uniform Algebras. J. Funct. Anal. 35 (1980), 64-108 
[Gr] Griffiths, Ph; Two theorems on extension of holomorphic mappings, Invent. Math 14 (1971), 27-62

[HP] Hubbard, J., Papadopol P; Superattractive fixed points in $\mathbb{C}^{n}$. Preprint

[HS] Harvey, R., Shiffman, B; A characterization of holomorphic chains. Ann. Math. 99 (1974), 553-587

[H] Hörmander, L; The analysis of linear partial differential operators 1. Springer, Berlin Heidelberg New York (1983)

[Lel] Lelong, P; Fonctions plurisousharmoniques et formes differentielles positives. Gordon and Breach, Dunod, Paris (1968)

[Le2] Lelong, P; Discontinuite et annulation de l'operateur de Monge Ampere complexe. In: Lecture Notes Math. Springer, Berlin Heidelberg New York 1028 (1983), 217-224

[LG] Lelong, P., Gruman, L; Entire Functions of several variables. Grundlehren Math. Wiss. Springer, Berlin Heidelberg New York 282 (1986)

[Ok] Oka, K; Sur les fonctions analytiques de plusieurs variables, IX. Une Mode Nouvelle engeandrant les Domaines Pseudoconvexes. Jap. J. Math. 32 (1962), 1-12

[Ri] Riemenschneider, $O$; Uber den Flacheneinhalt analytischen Mengen und die Erzeugung $k$-pseudokonvexe Gebiete. Invent. Math. 2 (1967), 307-331

[Ro] Ronkin, L.I; Weak convergence of the currents $\left(d d^{\prime} u\right)^{2}$ and asymptotics of order functions for holomorphic mappings of regular growth. Sib. Math. Zh. 25 (146) (1984) 645-650

[Ru] Ruelle, D; Elements of differentiable Dynamics and bifurcation theory. Acad. Press (1989)

[Ro] Rothstein, W; Ein neuer Beweis das Hartogschen Hauptsatzes und seine Ausdehnung auf meromorphe Funktionen. Math. Z. 53 (1950) 84-95

[Si] Sibony, N; Quelques problems de prolongement de courants en Analyse complexe. Duke Math. J. 52 (1985), 157-197

[Siu1] Siu, Y.T; Extension of meromorphic maps into Kahler manifolds. Ann. Math. 102 (1975) 421-462

[Siu2] Siu, Y.T; Techniques of extension of analytic objects. Marcel Dekker, New York (1974) 